\title{
Mecanismos fisiopatológicos, neurohormonales y objetivos terapéuticos en la insuficiencia cardiaca aguda
}

\author{
Christian Osmar Chávez-Alfonso¹, *Osmar Antonio Centurión ${ }^{1,2}$ \\ ${ }^{1}$ Universidad Nacional de Asunción, Hospital de Clínicas, División de Medicina Cardiovascular. San Lorenzo, \\ Paraguay \\ ${ }^{2}$ Sanatorio Metropolitano, Departamento de Investigación en Ciencias de la Salud. Fernando de la Mora. Paraguay
}

Cómo referenciar este artículo/

How to reference this article:
Chávez-Alfonso co, Centurión OA. Mecanismos fisiopatológicos, neurohormonales y objetivos terapéuticos en la insuficiencia cardiaca aguda. Mem. Inst. Investig. Cienc. Salud. 2019; 17(3): 82-96

\section{RE S U M E N}

La insuficiencia cardiaca aguda (ICA) se define como la aparición rápida o el empeoramiento de los síntomas o signos de IC. Es una entidad potencialmente mortal que requiere evaluación y tratamiento urgentes y típicamente conlleva la hospitalización urgente. La descompensación de la insuficiencia cardiaca crónica puede ocurrir sin que existan factores desencadenantes conocidos. Se produce una disfunción endotelial que puede deberse a un desequilibrio en el medio neuro-hormonal, inflamatorio, y oxidativo en la circulación y en las células endoteliales. Se produce clínicamente hipoperfusión miocárdica, reducción del flujo coronario, disfunción isquémica, aumento de la rigidez vascular y deterioro de la distensibilidad arterial que agravan en mayor medida la lesión miocárdica. La evaluación inicial y la monitorización no invasiva continua de las funciones vitales cardiorrespiratorias son esenciales. Los diuréticos son la piedra angular del tratamiento de los pacientes con ICA y signos de sobrecarga de fluidos y congestión. La terapia de reemplazo renal se debe reservar para pacientes que no responden al tratamiento con diuréticos. Los vasodilatadores intravenosos son los segundos fármacos más utilizados en la ICA para el alivio de los síntomas; sin embargo, no existen pruebas definitivas que confirmen su efecto beneficioso. El uso de inotrópicos debería estar reservado a los pacientes con disfunción sistólica que afecta a la perfusión de órganos vitales. Los dispositivos de asistencia ventricular y otras formas de asistencia mecánica circulatoria pueden emplearse como tratamiento puente en pacientes seleccionados.

Palabras clave: Insuficiencia cardiaca aguda, fisiopatológía, manejo terapéutico.

\section{Physiopathological, neurohormonal and therapeutic targets in acute heart failure}

\section{A B S T R A C T}

Acute heart failure (AHF) is defined as the rapid onset or worsening of the symptoms or signs of heart failure. It is a potentially fatal entity that requires urgent evaluation and treatment and typically involves urgent hospitalization. Decompensation of chronic heart failure can occur without known triggers. An endothelial dysfunction occurs that may be due to an imbalance in the neuro-hormonal, inflammatory, and oxidative environment in the circulation and in the endothelial cells. Myocardial hypoperfusion is clinically produced, coronary flow reduction, ischemic dysfunction, increased vascular rigidity and deterioration of arterial compliance that aggravate myocardial injury to a greater extent. Initial evaluation and continuous non-invasive monitoring of vital cardiorespiratory functions are essential. Diuretics are the cornerstone of the treatment of patients with AHF and signs of fluid overload and congestion. Renal replacement therapy should be reserved for patients 
who do not respond to treatment with diuretics. Intravenous vasodilators are the second most used drugs in the AHF for the relief of symptoms; however, there is no definitive evidence to confirm its beneficial effect. The use of inotropics should be reserved for patients with systolic dysfunction that affects the perfusion of vital organs. Ventricular assist devices and other forms of mechanical circulatory assistance can be used as a bridge treatment in selected patients.

Keywords: Acute heart failure, pathophysiology, therapeutic management.

\section{INTRODUCCIÓN}

La insuficiencia cardiaca (IC) es un síndrome clínico caracterizado por síntomas típicos como disnea, edema y fatiga, que puede ir acompañado de signos como presión venosa yugular elevada, crepitantes pulmonares y edema periférico causados por una anomalía cardiaca estructural o funcional que producen una reducción del gasto cardiaco o una elevación de las presiones intracardiacas en reposo o en estrés ${ }^{(1,2)}$. La insuficiencia cardíaca aguda (ICA) se define como un deterioro rápido de los signos y síntomas de insuficiencia cardiaca y que necesitan tratamiento urgente ${ }^{(3,4)}$. La ICA es un síndrome clínico cuya fisiopatología es compleja y que aún no se conoce por completo. Dada la diversidad de formas de presentación clínica, intervienen en ella varios mecanismos fisiopatológicos diferentes, junto con factores que desencadenan una descompensación circulatoria. Los factores que desencadenan la ICA pueden ser isquemia, hipertensión, arritmias, comorbilidades no cardiacas, y fármacos ${ }^{(4)}$.

Hasta un $15-20 \%$ de los pacientes ingresados por síndrome coronario agudo presentan signos y síntomas de IC y en otro $10 \%$ se produce IC durante la estancia hospitalaria; la incidencia es aún mayor en los estudios que se centran en pacientes con diagnóstico de ICA, de los que hasta un $40 \%$ puede haber sufrido un síndrome coronario como factor desencadenante. Tiene interés señalar que actualmente el SCA complicado por ICA a menudo se considera una entidad clínica distinta, caracterizada por interacciones estructurales, hemodinámicas y neurohormonal complejas, que hacen necesaria la derivación urgente a intervención coronaria y se asocian a mala evolución y peor pronóstico comparada con la ICA desencadenadas por otras causas ${ }^{(4)}$.

La IC continúa siendo una causa importante de morbilidad y mortalidad en todo el mundo ${ }^{(5-7)}$, y una historia de enfermedad coronaria está presente en más de la mitad de los pacientes con ICA ingresados en el hospital ${ }^{(8-11)}$. El síndrome coronario agudo complicado con ICA conduce a un aumento de varias veces en la mortalidad hospitalaria en comparación con aquellos sin ICA. Además, una proporción sustancial de pacientes con enfermedad coronaria hospitalizados desarrollan ICA durante la estancia hospitalaria, y tiene un pronóstico peor que los que se presentan inicialmente con ICA ${ }^{(12)}$. Es así que la ICA representa un síndrome que tiene una fisiopatología heterogénea con resultados variables. Por lo tanto, es nuestra intención realizar un análisis de los mecanismos fisiopatológicos, neurohormonales y de los objetivos terapéuticos en la insuficiencia cardiaca aguda.

\section{Factores desencadenantes de la insuficiencia cardiaca aguda}

La ICA se define como la aparición rápida o el empeoramiento de los síntomas o signos de IC. Es una entidad potencialmente mortal que requiere evaluación y tratamiento urgentes y típicamente conlleva la hospitalización urgente. La ICA se puede presentar como primera manifestación de la IC, o de nueva aparición o de novo, y más frecuentemente, como consecuencia de una descompensación aguda de la IC crónica o descompensada, que puede estar causada por una disfunción cardiaca primaria o precipitada por factores extrínsecos.

La disfunción miocárdica aguda, ya sea de origen isquémica, inflamatoria o tóxica, además de la insuficiencia valvular aguda o el taponamiento pericárdico son las más frecuentes causas cardiacas primarias de la ICA. La descompensación de la insuficiencia cardiaca crónica puede ocurrir sin que existan factores desencadenantes conocidos, pero generalmente están presentes uno o más factores, como la infección, la hipertensión no controlada, alteraciones del ritmo o la falta de adherencia al tratamiento o la $\operatorname{dieta}^{(1-4)}$. En la Tabla 1 se pueden observar las principales causas desencadenantes de insuficiencia cardiaca aguda. 
Tabla 1: Factores desencadenantes de la insuficiencia cardiaca aguda

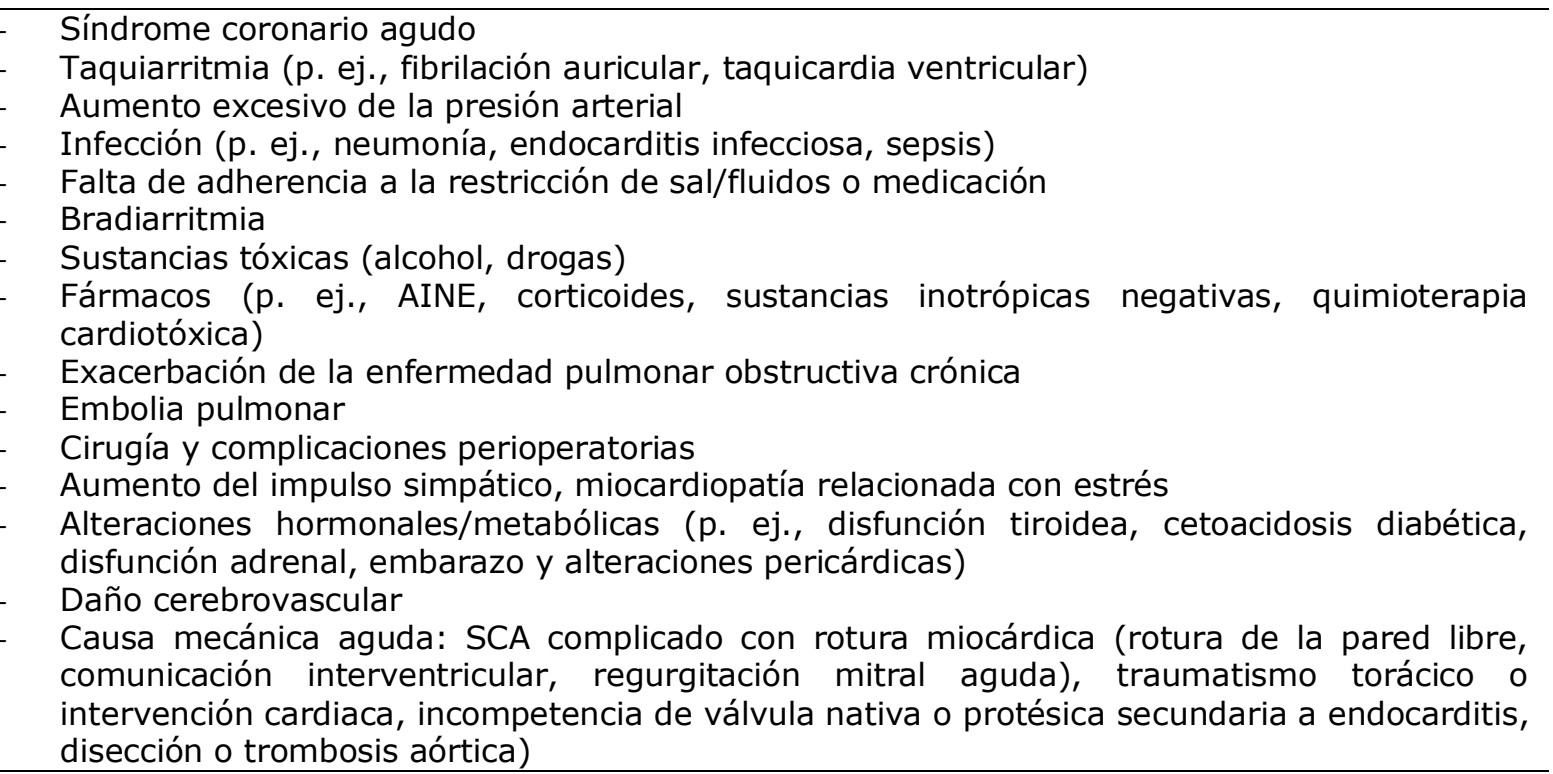

En la mayoría de los casos clínicos, los pacientes con ICA presentan una presión arterial sistólica normal (90-140 mmHg) o elevada (> $140 \mathrm{mmHg}$; ICA hipertensiva). Solo el 5-8\% de los pacientes presentan una presión arterial sistólica baja (< $90 \mathrm{mmHg}$; ICA hipotensiva), la cual se asocia con un mal pronóstico, especialmente en presencia de hipoperfusión. Otra estrategia consiste en clasificar a los pacientes según la presencia de las siguientes causas o factores desencadenantes de la descompensación, que requieren tratamiento o corrección urgente para su resolución: SCA, emergencia hipertensiva, arritmias rápidas o bradicardia/alteraciones del ritmo graves, causa mecánica aguda subyacente a la ICA o embolia pulmonar aguda(13-16). Muchos pacientes con IC y miocardiopatía isquémica tienen antecedentes de infarto de miocardio o revascularización. Sin embargo, una coronariografía normal no excluye la presencia de tejido miocárdico cicatricial o afección de la microcirculación coronaria, como evidencia alternativa de la cardiopatía isquémica.

En los últimos 30 años, los avances en los tratamientos y su implementación han mejorado la supervivencia y han reducido la tasa de hospitalización en pacientes con IC con fracción de eyección reducida (IC-FEr). Los datos europeos más recientes demuestran que las tasas de mortalidad por todas las causas a los 12 meses de los pacientes hospitalizados - estables/ambulatorios con IC son del 17 y el $7 \%$ respectivamente. Las tasas de hospitalización a los 12 meses fueron del 23,6\% para la ICA $^{(17)}$. En pacientes con IC (hospitalizados o ambulatorios), la mayoría de las muertes se deben a causas cardiovasculares, fundamentalmente muerte súbita y empeoramiento de la IC. La mortalidad por todas las causas suele ser mayor en la IC-FEr que en la insuficiencia cardiaca con fracción de eyección conservada (IC-FEc). Las hospitalizaciones se deben frecuentemente a causas no cardiovasculares, especialmente en pacientes con HF-FEc ${ }^{(16)}$.

Mecanismos fisiopatológícos de la insuficiencia cardiaca aguda

Los mecanismos fisiopatológicos que se describen para la insuficiencia cardiaca aguda se encuentran resumidos e ilustrados en la Figura 1. 


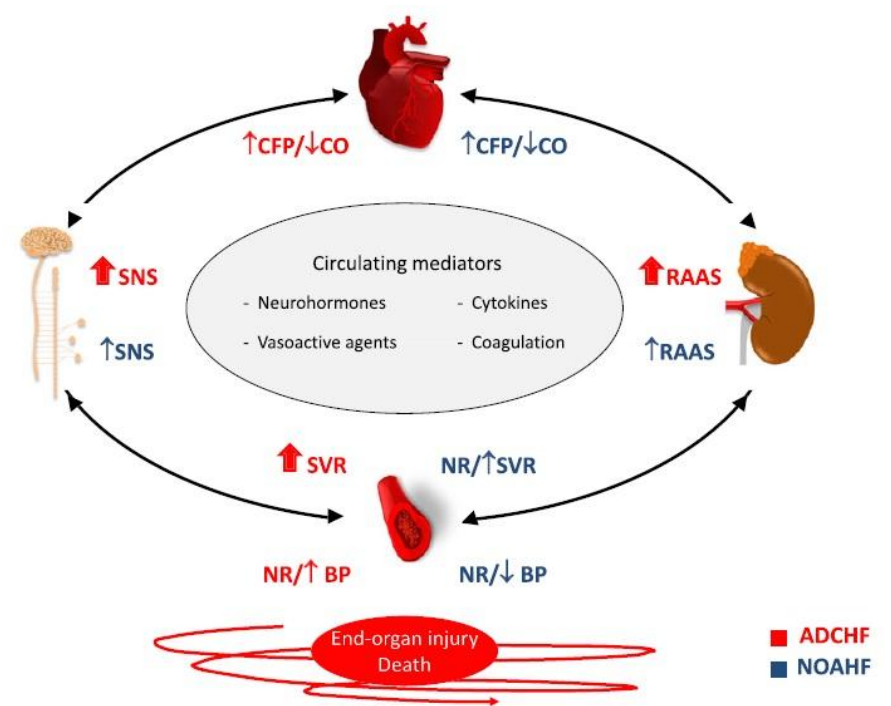

Figura 1: Fisiopatología de la insuficiencia cardiaca aguda.

ADCHF: Descompensación aguda de la insuficiencia cardiac crónica. BP: Presión sanguínea. CFP: Presión de llenado cardiaco. CO: Gasto cardiac. NR: Rango normal.

NOAHF: Insuficiencia cardiac aguda de inicio nuevo. RAAS: Sistema renina-angiotensinaaldosterone. SNS: Sistema nervioso simpatico. SVR: Resistencia vascular sistémica. Reimpreso con permiso de ${ }^{(37)}$ Ferreira J. Vascular phenotypes of acute decompensated vs. new-onset heart failure: treatment implications. ESC Heart Failure 2017;4:679-685.

Activación neurohormonal: Es un fenómeno que se describe para la descompensación circulatoria. Es detectable en el tejido miocárdico y en otros órganos afectados, y debido a su carácter generalizado, también puede identificarse en la circulación periférica. Su papel durante el estrés hemodinámico es principalmente adaptativo, puesto que permiten combatir el mayor esfuerzo realizado por el corazón y el sistema circulatorio, pero tan solo durante un plazo corto. Si se mantiene por cierto tiempo, pasa a ser una mala adaptación y a tener un carácter nocivo, lo cual aumenta la insuficiencia circulatoria y deteriora la homeostasis generalizada. Intervienen en la progresión de la disfunción cardiaca, tanto durante la fase aguda de la descompensación circulatoria como después de ella, puesto que su influencia va mucho más allá del episodio de ICA y contribuye a producir una progresión constante de la IC crónica. Son potentes factores predictivos de mala evolución y pronostican un aumento de la mortalidad a corto y largo plazo, así como un aumento del riesgo de hospitalizaciones repetidas a causa de episodios de ICA posteriores ${ }^{(4-7)}$.

La activación neurohormonal incluye la activación de los siguientes sistemas y vías de señalización relacionados: 1) Sistema renina-angiotensina-aldosterona; 2) sistema nervioso simpático (con la depleción del sistema nervioso parasimpático y el control reflejo cardiopulmonar anormal asociado, es decir, una atenuación del barorreflejo y un aumento de los quimiorreflejos centrales y periféricos); 3) arginina-vasopresina (junto con la copeptina, el segmento carboxiterminal de la pre-pro-vasopresina como indicador indirecto estable y fiable de la vasopresina); 4) endotelina; 5) adrenomedulina, y 6) el sistema de péptidos natriuréticos ${ }^{(1-4)}$.

Activación inflamatoria: La reacción inflamatoria incluye predominantemente activación de la respuesta inmunitaria innata, aumento de la expresión de mediadores proinflamatorios (como factor de necrosis humoral, interleucina 1, interleucina 6 y ST2), activación del sistema del complemento, producción de anticuerpos y sobreexpresión de moléculas del complejo mayor de histocompatibilidad, así como moléculas de adhesión ${ }^{(4-9)}$.

Estrés oxidativo: El estrés oxidativo se asocia a un exceso de especies moleculares de oxígeno reactivas que, por ejemplo, reaccionan con el óxido nítrico, alteran la señalización fisiológica y conducen a la producción de moléculas tóxicas y reactivas (peroxinitrito, isoprostano, aminotioles) y aumentan el catabolismo de las purinas, lo cual incrementa a su vez la actividad de la xantina oxidasa y, por consiguiente, las concentraciones séricas de ácido úrico e induce también un aumento de la liberación de mieloperoxidasa por los neutrófilos y monocitos activados ${ }^{(4)}$.

Disfunción miocárdica: la descompensación circulatoria se produce siempre en los pacientes con función miocárdica anormal, pero la disfunción cardiaca observada en los 
pacientes con ICA varía en su carácter (disfunción sistólica/diastólica, corazón izquierdo/derecho), el factor desencadenante (isquemia, inflamación, hipertensión) y el curso clínico (empeoramiento rápido o gradual) ${ }^{(12)}$.

La función sistólica del ventrículo izquierdo puede variar entre una función normal y un deterioro grave, y puede acompañarse de disfunción diastólica o insuficiencia mitral. Un problema clínico importante es la disfunción ventricular derecha, que suele complicar la disfunción del corazón izquierdo. Todas estas anomalías afectan a los síntomas de la ICA y se asocian a una evolución clínica desfavorable. Las anomalías observadas en el miocardio durante la ICA muy probablemente se deban a los fenómenos citados previamente. Están relacionadas con la progresión de la disfunción miocárdica y las anomalías estructurales asociadas a ello, como la hipertrofia miocardiocitaria, la apoptosis miocardiocitaria, la depresión de la contractilidad miocárdica, la inhibición de la capacidad de respuesta de los miocitos a la estimulación beta adrenérgica, el crecimiento de fibroblastos, la fibrosis y el remodelado, por mencionar tan solo unas pocas. Sean cuales fueren los mecanismos moleculares subyacentes y los factores desencadenantes, se ha propuesto que los episodios de ICA se asocian a una notable pérdida de miocitos (necrosis) y a alteraciones dinámicas en la arquitectura de la matriz extracelular miocárdica (remodelado). El daño sufrido por los miocitos puede reflejarse en la confirmación de unas concentraciones elevadas de troponinas cardiacas circulantes ${ }^{(12-15)}$.

Disfunción endotelial: la ICA se caracteriza también por una disfunción endotelial generalizada. Esta disfunción puede deberse a un desequilibrio en el medio neurohormonal, inflamatorio y oxidativo en la circulación y en las células endoteliales, así como a otros factores no identificados, que pueden causar clínicamente: a) hipoperfusión miocárdica, reducción del flujo coronario y disfunción isquémica; b) aumento de la rigidez vascular y deterioro de la distensibilidad arterial que agravan en mayor medida la lesión miocárdica; c) vasoconstricción en la circulación sistémica y pulmonar, que da lugar a un aumento de la sobrecarga ventricular izquierda y derecha; d) aumento secundario de la estimulación simpática asociado a la endelina y liberación de catecolaminas, y e) disfunción renal, que se refleja principalmente en la reducción de la excreción de sodio, pero se asocia también a otras anomalías ${ }^{(3,4)}$.

Otras disfunciones de órganos: es importante señalar que la disfunción cardiaca en sí es tan solo uno de los elementos que forman parte de la compleja fisiopatología de la ICA, y que otras anomalías de los vasos sanguíneos y mecanismos patogénicos periféricos que afectan a otros órganos del cuerpo (como los riñones, el hígado, el endotelio y los pulmones) desempeñan un papel crucial (si no dominante en todos los casos). La disfunción renal tiene un papel importante en la fisiopatología de la ICA, pero su origen no se conoce por completo. La disfunción renal incluye disminución del filtrado glomerular, función tubular anormal (que se refleja en unas concentraciones elevadas de lipocalina asociada a la gelatinasa neutrofílica, molécula de lesión renal tanto en sangre periférica como en orina) y actividad endocrina insuficiente (secreción inadecuada de eritropoyetina y renina). Se ha señalado que la disfunción renal puede deberse a una activación neurohormonal generalizada, inflamación, estrés oxidativo, deterioro de la hemodinámica intrarrenal como consecuencia de una hemodinámica extrarrenal anormal que afecta al flujo sanguíneo y las presiones renales o de una alteración de los mecanismos de regulación hemodinámica intrarrenal, una nefropatía intrínseca y causas iatrogénicas ${ }^{(16-19)}$.

Respuesta hemodinámica: la ICA se asocia a una amplia variedad de respuestas hemodinámicas anormales, entre las que se encuentran la reducción característica del gasto cardiaco, el aumento de las presiones de llenado y el incremento de la postcarga. Los mecanismos patogénicos que conducen directamente a la descompensación circulatoria no se conocen por completo. Aunque la congestión pulmonar y/o periférica continúa siendo una característica prominente de la ICA, es importante señalar que tan solo aproximadamente un $50 \%$ de los pacientes sufren un aumento significativo del peso corporal asociado a una acumulación real de líquidos. Existen dos conceptos en los que los mecanismos patogénicos que subyacen a las congestiones difieren, es decir, la acumulación de líquido exógeno frente al desplazamiento de líquido endógeno procedente de los reservorios venosos del organismo. La primera teoría es más convencional y parte del supuesto de que la congestión se debe a la retención de sodio y agua, que se instaura de manera gradual y se asocia a una acumulación de líquido exógeno, aumento del peso corporal, aumento del volumen circulatorio efectivo y disfunción renal prominente. La segunda teoría se basa en la 
hipótesis de que la congestión es consecuencia de un desplazamiento de líquido endógeno. Este se instaura de forma rápida, se asocia principalmente a un aumento de la estimulación simpática que causa vasoconstricción en los vasos de capacitancia venosos y conduce a un desplazamiento del volumen del reservorio de sangre venosa hacia la circulación sistémica. No se asocia a acumulación de líquido exógeno adicional ni a aumento del peso corporal. Este último mecanismo forma parte de los mecanismos adaptativos de conservación, y se opone al ortostatismo, a la vez que proporciona un volumen circulatorio de precarga adicional en respuesta a la bipedestación, el ejercicio, el estrés y los traumatismos ${ }^{(4,5)}$.

En los pacientes con IC que tienen relaciones de presión-volumen anormales, un desplazamiento de volumen, incluso relativamente pequeño, a través de un mecanismo simpático puede elevar inadecuadamente las presiones pulmonares y causar extravasación de líquido y congestión. Los factores que desencadenan la activación simpática aguda relativa pueden ser diferentes. El aumento de la actividad tónica y episódica de los quimiorreceptores sin acumulación evidente de agua y sodio puede ser atribuible a los episodios rápidos de $\operatorname{ICA}^{(6)}$.

\section{Diagnóstico y evaluación clínica inicial de la ICA}

El proceso diagnóstico se debe iniciar en el contexto pre-hospitalario y continuar en el servicio de urgencias para establecer el diagnóstico e iniciar el tratamiento adecuado lo antes posible. El beneficio del tratamiento precoz en los SCA está bien establecido y se debe considerar en el contexto de la ICA. En paralelo, se debe identificar y manejar las entidades potencialmente mortales $o$ factores desencadenantes coexistentes que requieren tratamiento o corrección urgente. Normalmente, un paso inicial del proceso diagnóstico de la ICA es descartar otras causas alternativas de los síntomas y signos del paciente tales como infección pulmonar, anemia grave, e insuficiencia renal aguda ${ }^{(20-26)}$.

Cuando se confirma el diagnóstico de ICA es imprescindible realizar una evaluación clínica para decidir el posterior tratamiento. Se recomienda que el diagnóstico inicial de ICA se base en una minuciosa historia clínica para valorar los síntomas, la historia cardiovascular previa y los desencadenantes potenciales, cardiacos y no cardiacos, así como en la evaluación de los signos/síntomas de congestión o hipoperfusión mediante exploración física, y se confirme posteriormente mediante pruebas adicionales, como ECG, radiografía torácica, pruebas de laboratorio con biomarcadores específicos y ecocardiografía. Para los pacientes que se presentan con ICA, el inicio precoz del tratamiento adecuado es de suma importancia. Típicamente, los síntomas y signos de ICA reflejan sobrecarga de fluidos con congestión pulmonar o edema periférico o, gasto cardiaco reducido con hipoperfusión periférica.

La radiografía torácica: puede ser una prueba útil para el diagnóstico de la ICA. La congestión venosa pulmonar, el derrame pleural, el edema intersticial o alveolar y la cardiomegalia suelen ser los hallazgos más específicos de la ICA, aunque en hasta un $20 \%$ de los pacientes con ICA la radiografía torácica es prácticamente normal. La radiografía torácica también es útil para identificar causas alternativas no cardiacas que pueden causar o contribuir a los síntomas, tales como, neumonía, infecciones pulmonares no consolidadas.

El electrocardiograma: rara vez es normal en la ICA (valor predictivo negativo alto). También es útil para identificar enfermedades cardiacas subyacentes y factores desencadenantes potenciales como la FA rápida, la isquemia miocárdica aguda.

La ecocardiografía: de forma inmediata solo es imprescindible en pacientes con inestabilidad hemodinámica y en pacientes con sospecha de alteraciones cardiacas estructurales 0 funcionales que pueden ser potencialmente mortales como las complicaciones mecánicas, regurgitación valvular aguda, y disección aórtica. Se debe considerar la ecocardiografía precoz para todos los pacientes con ICA de novo o con función cardiaca desconocida. Normalmente no es necesario repetir la ecocardiografía, excepto cuando el estado clínico del paciente se deteriora significativamente.

\section{Pruebas de laboratorio:}

Péptidos natriuréticos: Tras el ingreso en el servicio de urgencias o unidad de cuidados intensivos o coronarios, se determinará la concentración plasmática de los péptidos natriuréticos (PN), el péptido natriurético tipo B (BNP), y el (NT-proBNP o MR-proANP) de todos los pacientes con disnea aguda y sospecha de ICA para diferenciar la disnea aguda causada por ICA o por otras causas no cardiacas. Los BNP y NT-proBNP tienen una sensibilidad y una especificidad bastante alta (Figuras 2 y Figura 3 ). En pacientes con valores de PN normales y sospecha de ICA, este diagnóstico es improbable (umbrales: BNP 
$<100$ pg/ml, NT-pro- BNP $<300$ pg/ml, MR-proANP $<120$ pg/ml). No obstante, las cifras de PN elevadas no confirman automáticamente el diagnóstico de ICA, ya que pueden darse por numerosas causas cardiacas y no cardiacas como se ejemplifican en la Tabla 2.

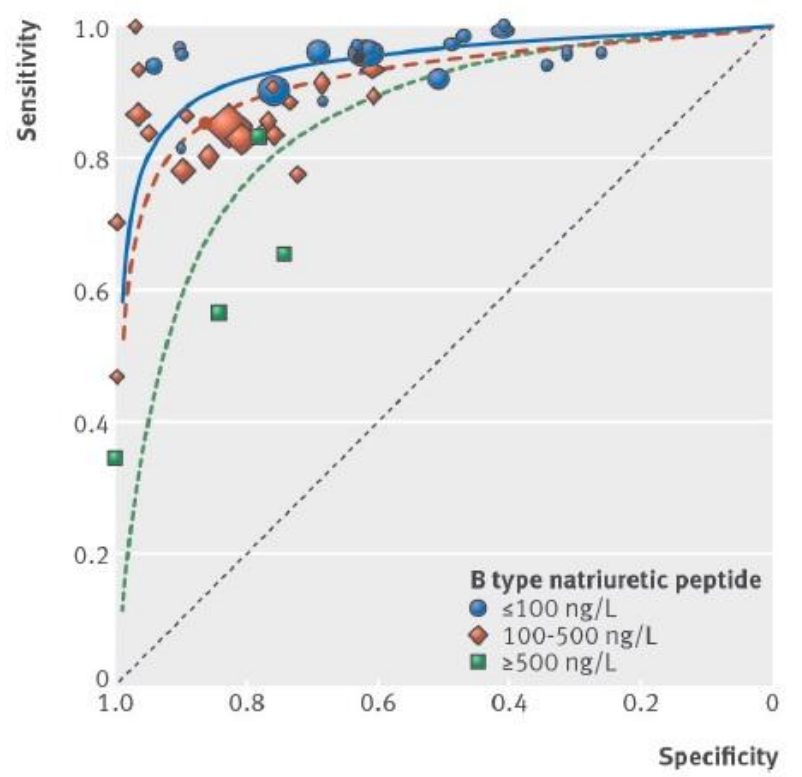

Figura 2: Se observan los resultados de especificidad y sensibilidad de los Resultados con BNP. Los datos están separados de acuerdo al umbral de nivel sérico de BNP obtenido. Reimpreso con permiso de (22) Roberts E, Ludman AJ, Dworzynski K, Al-Mohammad A, Cowie MR, McMurray JJV, et al. The diagnostic accuracy of the natriuretic peptides in heart failure: systematic review and diagnostic metaanalysis in the acute care setting. BMJ 2015;350:h910 doi: 10.1136/bmj.h910.

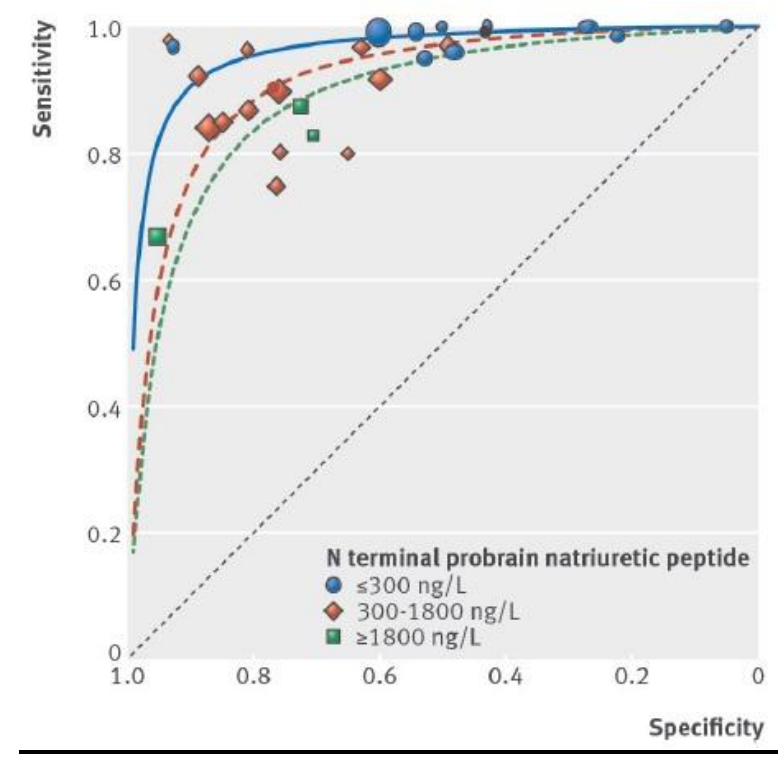

Figura 3: Se observan los resultados de especificidad y sensibilidad de los Resultados con NT-proBNP. Los datos están separados de acuerdo al umbral de nivel sérico de NT-proBNP obtenido. Reimpreso con permiso de ${ }^{(22)}$ Roberts E, Ludman AJ, Dworzynski K, Al-Mohammad A, Cowie MR, McMurray JJV, et al. The diagnostic accuracy of the natriuretic peptides in heart failure: systematic review and diagnostic meta-analysis in the acute care setting. BMJ 2015;350: h910 doi: 10.1136/bmj.h910.

Tabla 2: Causas de aumento de los péptidos natriuréticos

\begin{tabular}{ll}
\hline Cardiacas & No cardiacas \\
\hline Insuficiencia cardiaca & Edad avanzada \\
Síndromes coronarios agudos & Ictus isquémico \\
Embolia pulmonar & Hemorragia subaracnoidea \\
Miocarditis & Disfunción renal \\
Hipertrofia ventricular izquierda & Disfunción hepática (fundamentalmente \\
\hline
\end{tabular}


Miocardiopatía hipertrófica o restrictiva Valvulopatías

Cardiopatía congénita

Taquiarritmias auriculares y ventriculares Contusión cardiaca

Cardioversión, shock por DAI

Procedimientos quirúrgicos que implican al corazón

Hipertensión pulmonar cirrosis hepática con ascitis)

Síndrome paraneoplásico

Enfermedad pulmonar obstructiva crónica

Infecciones graves (incluidas neumonía y sepsis)

Quemaduras graves

Anemia

Otras pruebas de laboratorio al ingreso: Se deben realizar las siguientes pruebas de laboratorio al ingreso de todos los pacientes con ICA: troponina cardiaca, nitrógeno ureico en sangre (BUN), creatinina, electrolitos (sodio, potasio), pruebas de función hepática, tirotropina (TSH), glucosa y recuento sanguíneo completo; la prueba de dímero $\mathrm{D}$ está indicada para pacientes con sospecha de embolia pulmonar aguda. La determinación sistemática de gases arteriales no es necesaria y se debe restringir a los pacientes cuya oxigenación no se puede evaluar rápidamente con oximetría de pulso. Sin embargo, puede ser útil cuando sea preciso medir las presiones parciales de $\mathrm{O} 2$ y $\mathrm{CO} 2$. Cabe destacar que la determinación de troponinas cardiacas es útil para la detección de SCA como causa subyacente de la ICA. Sin embargo, en la gran mayoría de los pacientes con ICA se detectan altas concentraciones de troponinas circulantes, frecuentemente sin isquemia miocárdica obvia ni una complicación coronaria aguda, lo cual indica la presencia de daño o necrosis de miocitos en estos pacientes. También en pacientes con embolia pulmonar aguda como causa subyacente de la descompensación, la determinación de altas concentraciones de troponinas es útil para la estratificación del riesgo y la toma de decisiones ${ }^{(19-21)}$.

Se recomienda medir la creatinina, BUN y electrolitos cada 1-2 días durante la hospitalización y antes del alta. No obstante, la gravedad del caso podría justificar una mayor frecuencia de las determinaciones. Se puede considerar la determinación de PN antes del alta para la evaluación pronóstica. También la evaluación de la concentración de procalcitonina en pacientes con ICA y sospecha de infección coexistente puede ser considerada, especialmente para el diagnóstico diferencial de la neumonía y guiar el tratamiento antibiótico si se considera necesario. La función hepática suele estar afectada en los pacientes con ICA debido a alteraciones hemodinámicas. Los resultados anormales en las pruebas de la función hepática permiten identificar a los pacientes con mayor riesgo de un pronóstico desfavorable y pueden ser útiles para optimizar el tratamiento ${ }^{(27-29)}$.

Dado que el hipotiroidismo y el hipertiroidismo pueden precipitar la ICA, se debe determinar la TSH de los pacientes con ICA de nuevo diagnóstico. La evaluación hemodinámica invasiva sistemática con catéter arterial pulmonar no está indicada para el diagnóstico de la ICA. Puede ser útil en casos seleccionados de pacientes hemodinámicamente inestables con un mecanismo de deterioro desconocido.

\section{Abordaje y manejo terapéutico de la ICA}

La ICA es una enfermedad potencialmente mortal, por lo que se debe trasladar al paciente rápidamente a un hospital, preferiblemente a un centro con servicio cardiológico intensivo. El diagnóstico precoz es importante en la ICA, por ello, el proceso diagnóstico y el tratamiento farmacológico y no farmacológico se iniciarán lo antes posible y en paralelo en todos los pacientes con sospecha de ICA. La evaluación inicial y la monitorización no invasiva continua de las funciones vitales cardiorrespiratorias, que incluye oximetría de pulso, presión sanguínea, frecuencia respiratoria y ECG inmediato y continuo, son esenciales para evaluar si la ventilación, la perfusión periférica, la oxigenación, la frecuencia cardiaca y la PA son adecuadas. También se debe monitorizar la diuresis, aunque no está recomendado el cateterismo urinario por sistema. Los pacientes con trastorno/insuficiencia respiratoria o deterioro hemodinámico deben ser transferidos a una zona del hospital donde se pueda proporcionar apoyo respiratorio y cardiovascular inmediatamente.

El siguiente paso consiste en identificar los factores desencadenantes/ las causas principales que producen la descompensación, que se debe tratar urgentemente para evitar un mayor deterioro. Entre ellas se incluyen:

Síndrome coronario agudo: Se debe tratar a los pacientes con SCA según las guías de la AHA/ACC y/o de la ESC sobre SCA sin elevación del segmento ST (SCASEST) e infarto 
agudo de miocardio con elevación del segmento ST (IAMCEST) ${ }^{(20-23)}$. La coexistencia de estas 2 entidades clínicas (SCA e ICA) caracteriza a un grupo de pacientes de riesgo muy alto para los que se recomienda una estrategia invasiva inmediata $(<2 \mathrm{~h}$ desde el ingreso en el hospital para pacientes con IAMSEST, de modo similar que para el tratamiento del IAMCEST) con la intención de llevar a cabo la revascularización, independientemente de los hallazgos electrocardiográficos o de biomarcadores.

Emergencia hipertensiva: la ICA precipitada por un aumento rápido y excesivo de la presión arterial (PA) se manifiesta típicamente como edema pulmonar agudo. Se debe considerar la inmediata reducción de la PA como objetivo terapéutico prioritario y se aplicará tan pronto como sea posible. Está recomendada la reducción intensiva de la PA (alrededor del $25 \%$ durante las primeras horas y con precaución después) con vasodilatadores intravenosos combinados con diuréticos del asa ${ }^{(30-32)}$.

Arritmias rápidas 0 bradicardia/alteraciones de la conducción graves: Las alteraciones graves del ritmo cardiaco en pacientes con ICA y condiciones inestables se deben corregir urgentemente con tratamiento farmacológico, cardioversión eléctrica o marcapasos temporal. Si se cree que la arritmia auricular o ventricular contribuye al deterioro hemodinámico del paciente, se recomienda la cardioversión eléctrica para restaurar el ritmo sinusal y mejorar el estado clínico del paciente ${ }^{(32)}$.

Causa mecánica aguda subyacente a la ICA: Se puede presentar como una complicación mecánica del SCA (rotura de la pared libre, comunicación interventricular, regurgitación mitral aguda), traumatismo torácico 0 intervención cardiaca o como incompetencia aguda de válvula nativa o protésica secundaria a endocarditis, disección o trombosis aórtica, además de causas de obstrucción raras (p. ej., tumores cardiacos). La ecocardiografía es fundamental para el diagnóstico; el tratamiento requiere normalmente apoyo circulatorio con intervención quirúrgica o percutánea.

Embolia pulmonar aguda: Cuando se confirma la embolia pulmonar aguda como la causa de shock o hipotensión, se recomienda el tratamiento de reperfusión primaria con trombólisis, intervención percutánea o embolectomía quirúrgica. A los pacientes con embolia pulmonar aguda se los debe tratar según las recomendaciones de las guías específicas $^{(24)}$.

La identificación de la etiología así como de los factores desencadenantes agudos se debe realizar en la fase inicial del tratamiento de la ICA (60-120 min) a fin de iniciar lo más precozmente posible la terapéutica específica sobre todo en aquellas entidades potencialmente mortales que requieren tratamiento o corrección urgente.

Criterios para la hospitalización: Los pacientes con disnea persistente y significativa o inestabilidad hemodinámica deben ser transferidos a una sala del hospital donde se pueda aplicar medidas de resucitación si fueran necesarias.

Para los pacientes de alto riesgo (disnea persistente y significativa, inestabilidad hemodinámica, arritmias recurrentes, ICA y SCA asociados), los cuidados iniciales se proporcionarán en una sala de alta dependencia (UCI/UCC).

Los criterios para el ingreso en la UCI/UCC incluyen cualquiera de los factores que podemos observar en la Tabla $3^{(25)}$. 
Tabla 3: Criterios para el ingreso en la unidad de cuidados intensivos cardiovasculares.

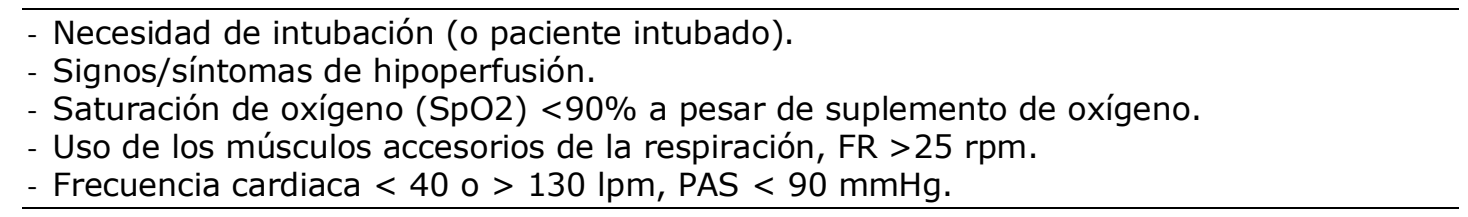

Los demás pacientes con ICA requieren el ingreso en una planta hospitalaria normal. Solo un pequeño porcentaje de pacientes con ICA ingresados en el servicio de urgencias (fundamentalmente por la exacerbación de los síntomas de IC con signos leves de congestión) reciben el alta directamente tras la administración de una pequeña dosis de diuréticos y el ajuste de las dosis del tratamiento médico y la recomendación de acudir a la consulta ambulatoria.

\section{Tratamiento en fase inicial de insuficiencia cardiaca aguda}

Tratamiento con oxígeno y apoyo ventilatorio: En la ICA no se debe utilizar sistemáticamente oxígeno en pacientes no hipoxémicos, ya que causa vasoconstricción y reducción del gasto cardiaco. En la EPOC, la hiperoxigenación puede aumentar el desajuste ventilación-perfusión, suprimir la ventilación y producir hipercapnia. Durante el tratamiento con oxígeno es preciso monitorizar el equilibrio acidobásico y el SpO2 transcutáneo ${ }^{(29-31)}$.

\section{Tratamiento farmacológico}

Diuréticos: Los diuréticos son la piedra angular del tratamiento de los pacientes con ICA y signos de sobrecarga de fluidos y congestión. Los diuréticos aumentan la secreción renal de agua y sal y además tienen cierto efecto vasodilatador. En pacientes con ICA y signos de hipoperfusión, se debe evitar la administración de diuréticos hasta que se logre una perfusión adecuada. La estrategia inicial para el tratamiento de la congestión incluye diuréticos intravenosos con la adición de vasodilatadores para el alivio de la disnea si la PA lo permite. Para aumentar la diuresis o superar la resistencia diurética, las opciones incluyen el bloqueo doble de la nefrona mediante diuréticos del asa (furosemida o torasemida) y tiacidas o dosis natriuréticas de ARM. Esta combinación requiere una monitorización minuciosa para evitar la hipopotasemia, la disfunción renal y la hipovolemia. Se debe limitar la dosis a la menor cantidad necesaria para lograr un efecto clínico adecuado y modificarla según la función renal y las dosis de diuréticos previas ${ }^{(27)}$.

Vasodilatadores: Los vasodilatadores intravenosos son los segundos fármacos más utilizados en la ICA para el alivio de los síntomas; sin embargo, no existen pruebas definitivas que confirmen su efecto beneficioso. Ejercen un efecto beneficioso doble al disminuir el tono venoso (que optimiza la precarga) y el tono arterial (que disminuye la postcarga), por lo que también pueden aumentar el volumen latido. Los vasodilatadores son especialmente útiles para los pacientes con ICA hipertensiva, mientras que los pacientes con PAS $<90 \mathrm{mmHg}$ (o con hipotensión sintomática) deben evitarlos. La dosis se debe controlar con cuidado para evitar una reducción excesiva de la PA, que se asocia con peores resultados. Los vasodilatadores deben ser empleados con precaución en pacientes con estenosis mitral o aórtica significativa ${ }^{(28,29)}$.

Inotrópicos: el uso de un inotrópico debería estar reservado a los pacientes con gasto cardiaco muy reducido que afecta a la perfusión de órganos vitales, lo cual ocurre más frecuentemente en la ICA hipotensiva. Los agentes inotrópicos no están recomendados para la ICA hipotensiva cuya causa subyacente sea la hipovolemia u otros factores potencialmente corregibles antes de la corrección de dichas causas. El levosimendán es preferible a la dobutamina para revertir el efecto de los bloqueadores beta si se cree que estos contribuyen a la hipoperfusión. Sin embargo, el levosimendán es un vasodilatador, por lo que no es adecuado para el tratamiento de pacientes con hipotensión (PAS < 85 $\mathrm{mmHg}$ ) o shock cardiogénico, excepto si se combina con otro inotrópico o vasopresor. Los inotrópicos, especialmente los que tienen mecanismos adrenérgicos, pueden causar taquicardia sinusal e inducir isquemia miocárdica y arritmias, por lo que es precisa la monitorización electrocardiográfica. Los inotrópicos deben emplearse con precaución, empezando por dosis relativamente bajas seguidas de un aumento gradual de la dosis bajo estrecha vigilancia ${ }^{(20)}$. 
Vasopresores: Los pacientes con hipotensión significativa son tratados con fármacos con potente acción vasoconstrictora arterial periférica, como la noradrenalina y la dopamina a dosis altas (> $5 \mu \mathrm{g} / \mathrm{kg} / \mathrm{min}$ ). Estos fármacos se administran para aumentar la PA y redistribuir el aporte sanguíneo hacia órganos vitales. No obstante, esto es a costa de un aumento de la postcarga del VI. La noradrenalina tiene menos efectos secundarios y una mortalidad más baja comparado con dopamina. La epinefrina (adrenalina) se debe reservar para pacientes con hipotensión persistente a pesar de presiones de llenado adecuadas y uso de otros fármacos vasoactivos, y también para protocolos de resucitación. Está también recomendada la profilaxis de las tromboembolias con heparina u otro anticoagulante, salvo que esté contraindicado o sea innecesario(21).

Digoxina: la digoxina está indicada fundamentalmente para pacientes con FA y frecuencia ventricular rápida ( $>110 \mathrm{lpm}$ ) y se administra en bolos intravenosos de 0,25-0,5 mg si no se administró previamente $(0,0625-0,125 \mathrm{mg}$ puede ser una dosis adecuada para pacientes con disfunción renal moderada o grave). Sin embargo, para los pacientes con comorbilidades $u$ otros factores que afecten al metabolismo de la digoxina (incluidos otros fármacos) o los pacientes mayores, es difícil establecer teóricamente la dosis de mantenimiento; en esta situación, la dosis se establecerá empíricamente según las concentraciones de digoxina en sangre periférica ${ }^{(22)}$.

Ultrafiltración extracorpórea: La mayoría de los pacientes que presentan insuficiencia cardíaca aguda muestran signos y síntomas de la sobrecarga de líquidos, que están estrechamente asociados con los resultados a corto y largo plazo. La ultrafiltración es una estrategia extremadamente atractiva para los pacientes con insuficiencia cardíaca aguda e importante retención de fluidos evidentes y concomitantes que no responde completamente a la terapia diurética ${ }^{(33)}$ (Figura 4 ).

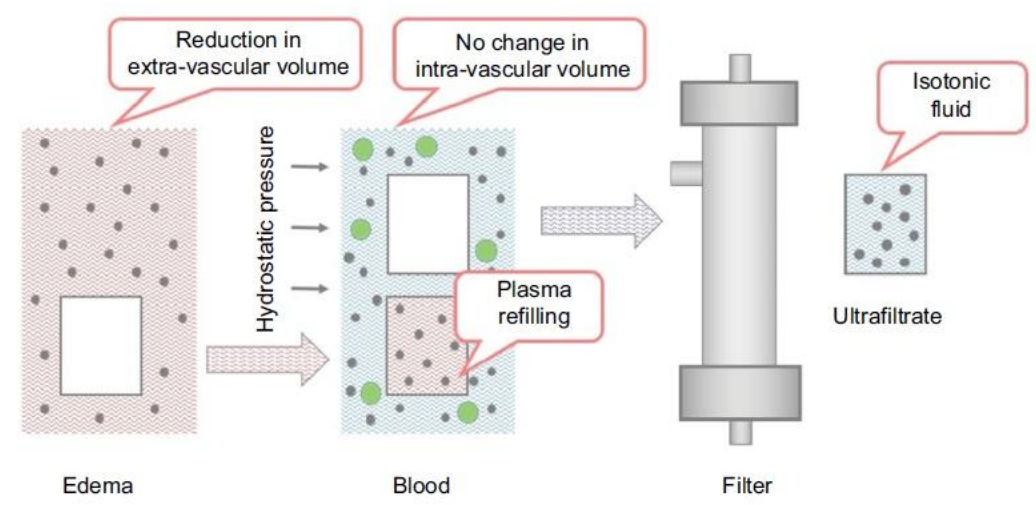

Proteins Small solutes $(<50,000 \mathrm{Da})$

Figura 4: Ejemplo esquemático del cambio de volumen del fluido entre el espacio extravascular y el intravascular durante la ultrafiltración. Reimpreso con permiso de ${ }^{(33)}$ Milazzo V, Cosentino N, Marenzi G. Extracorporeal ultrafiltration for acute heart failure: patient selection and perspectives. Vasc Health Risk Manag 2017;13: 449-456.

Los siguientes criterios pueden indicar la necesidad de terapia de reemplazo renal para pacientes con sobrecarga refractaria de volumen: oliguria que no responde a medidas de control de fluidos, hiperpotasemia grave $(\mathrm{K}+>6,5 \mathrm{mmol} / \mathrm{l})$, acidemia grave $(\mathrm{pH}<7,2)$, urea sérica $>25 \mathrm{mmol} /(150 \mathrm{mg} / \mathrm{dl})$ y creatinina sérica $>300 \mathrm{mmol} / \mathrm{l}(>3,4 \mathrm{mg} / \mathrm{dl})^{(23)}$.

Dispositivos de asistencia mecánica: Balón de contrapulsasión intraaórtico. Las indicaciones convencionales del balón de contrapulsación intraaórtico (BCIA) son el apoyo circulatorio antes de la corrección quirúrgica de problemas mecánicos agudos ( $p$. ej., rotura de comunicación interventricular y regurgitación mitral aguda), durante miocarditis aguda grave y en pacientes seleccionados con isquemia o infarto agudo de miocardio, durante y después de la revascularización percutánea o quirúrgica. No hay pruebas concluyentes de que el BCIA sea beneficioso en presencia de otras causas de shock cardiogénico ${ }^{(24,25)}$.

Dispositivos de asistencia ventricular: Los dispositivos de asistencia ventricular y otras formas de asistencia mecánica circulatoria pueden emplearse como tratamiento puente a la decisión o a más largo plazo para pacientes seleccionados ${ }^{(24,25)}$.

Otras intervenciones: Para pacientes con ICA y derrame pleural, se puede considerar la pleurocentesis con evacuación de fluidos, si fuera factible, para aliviar los síntomas de 
disnea. Para pacientes con ascitis, se puede considerar la paracentesis de líquido ascítico con evacuación de fluidos para aliviar los síntomas ${ }^{(23)}$.

Tratamiento de pacientes en shock cardiogénico: El shock cardiogénico se define como hipotensión (PAS $<90 \mathrm{mmHg}$ ) a pesar de un estado de llenado adecuado y signos de hipoperfusión. Los cuadros patogénicos de shock cardiogénico van desde la IC crónica, de bajo gasto y fase avanzada, a los episodios agudos de novo debidos frecuentemente al IAMCEST, pero también causados por otras etiologías diferentes del SCA. A todo paciente con sospecha de shock cardiogénico se le debe realizar inmediatamente ECG y ecocardiografía. Para pacientes con SCA complicado por shock cardiogénico, se recomienda realizar inmediatamente una coronariografía (durante las primeras 2 horas tras el ingreso) con intención de revascularizar al paciente. El objetivo del tratamiento farmacológico es mejorar la perfusión orgánica mediante el aumento del gasto cardiaco y la PA. Tras realizar una carga de fluidos, el tratamiento farmacológico consiste en la administración de un inotrópico y un fármaco vasopresor, según la necesidad del caso individual ${ }^{(34-38)}$.

Tratamientos orales basados en la evidencia: El tratamiento oral modificador de la enfermedad para la IC se debe mantener tras el ingreso por ICA, excepto en presencia de inestabilidad hemodinámica (hipotensión sintomática, hipoperfusión, bradicardia), hiperpotasemia o función renal muy deteriorada(39-44). En estos casos, la dosis diaria del tratamiento oral se puede reducir o interrumpir hasta que el paciente esté estabilizado ${ }^{(45-48)}$. El tratamiento con bloqueadores beta se puede mantener de manera segura durante la presentación de ICA, excepto en los casos de shock cardiogénico ${ }^{(49-52)}$. Un metanálisis reciente ha demostrado que la interrupción de beta bloqueantes a los pacientes hospitalizados por ICA se asoció con un aumento significativo de la mortalidad hospitalaria, la mortalidad a corto plazo y la variable combinada de rehospitalización a corto plazo o mortalidad $^{(26)}$.

\section{CONCLUSIÓN}

La insuficiencia cardiaca aguda es una entidad potencialmente mortal que requiere evaluación y tratamiento urgentes y típicamente conlleva la hospitalización de emergencia. Se produce una disfunción endotelial que puede deberse a un desequilibrio en el medio neuro-hormonal, inflamatorio, oxidativo en la circulación y en las células endoteliales. Se desencadena clínicamente hipoperfusión miocárdica, reducción del flujo coronario, disfunción isquémica, aumento de la rigidez vascular y deterioro de la distensibilidad arterial que agravan en mayor medida la lesión miocárdica.

Los diuréticos son la piedra angular del tratamiento de los pacientes con ICA y signos de sobrecarga de fluidos y congestión. Los diuréticos aumentan la secreción renal de agua y sal y además tienen cierto efecto vasodilatador. Se debe limitar la dosis a la menor cantidad necesaria para lograr un efecto clínico adecuado y modificarla según la función renal y las dosis de diuréticos previas. La terapia de reemplazo renal se debe reservar para pacientes que no responden al tratamiento con diuréticos. Los vasodilatadores intravenosos son los segundos fármacos más utilizados en la ICA para el alivio de los síntomas; sin embargo, no existen pruebas definitivas que confirmen su efecto beneficioso. El uso de inotrópicos debería estar reservado a los pacientes con gasto cardiaco muy reducido que afecta a la perfusión de órganos vitales, aunque no están recomendados para la ICA hipotensiva cuya causa subyacente sea la hipovolemia u otros factores potencialmente corregibles antes de la corrección de dichas causas. Los dispositivos de asistencia ventricular y otras formas de asistencia mecánica circulatoria pueden emplearse como tratamiento puente en pacientes seleccionados.

\section{REFERENCIAS BIBLIOGRÁFICAS}

1. Ponikowski $P$, Voors $A A$, Anker SD, Bueno $\mathrm{H}$, Cleland JG, Coats AJ, et al. 2016 ESC Guidelines for the Diagnosis and Treatment of Acute and Chronic Heart Failure. Rev Esp Cardiol (English ed). 2016; 69(12): 1167.

2. Yancy CW, Jessup M, Bozkurt B, Butler J., Casey DE Jr, Drazner MH, et al. 2013 ACCF/AHA guideline for the management of heart failure: executive summary: a report of the American College of Cardiology Foundation/American Heart Association Task Force on practice guidelines. Circulation. 2013;128 (16): 1810-52.

3. Martindale JL, Wakai A, Collins SP, Levy PD, Diercks D, Hiestand BC, et al. Diagnosing Acute Heart Failure in the Emergency Department: A Systematic 
Review and Meta-analysis. Academic Emergency Medicine 2016;23:223-42.

4. Ponikowski P, Jankowska E. Patogenia y presentación clínica de la insuficiencia cardiaca aguda. Rev Esp Cardiol. 2015; 68(4):331-37.

5. AlHabib KF, Elasfar AA, Alfaleh $H$, Kashour $T$, Hersi A, AlBackr $\mathrm{H}$, et al. Clinical features, management, and short- and long-term outcomes of patients with acute decompensated heart failure: phase I results of the HEARTS database. Eur ] Heart Fail. 2014;16(4):461-9.

6. Panduranga $P$, Sulaiman $K$, Al-Zakwani I, Alazzawi AA, Abraham A, Singh PP, et al. Demographics, Clinical Characteristics, Management, and Outcomes of Acute Heart Failure Patients: Observations from the Oman Acute Heart Failure Registry. Oman Med J. 2016;31(3):188-95.

7. Gysele S, Bleuminka AM, Knetscha MCJM, Sturkenboom SMJM, Strausa A, Hofmann JW, et al. Quantifying the heart failure epidemic: prevalence, incidence rate, lifetime risk and prognosis of heart failure The Rotterdam Study. European Heart Journal 2004; 25 (18): 1614-19.

8. Fonarow GC, Abraham WT, Albert NM, Gattis SW, Gheorghiade M, Greenberg BH, et al. OPTIMIZE-HF Investigators and Hospitals. Influence of a PerformanceImprovement Initiative on Quality of Care for Patients Hospitalized With Heart FailureResults of the Organized Program to Initiate Lifesaving Treatment in Hospitalized Patients With Heart Failure (OPTIMIZE-HF). Arch Intern Med. 2007;167(14):1493-502.

9. Parenica J, Spinar J, Vitovec J, Widimsky P, Linhart $A$, Fedorco $M$, et al. Long-term survival following acute heart failure: The Acute Heart Failure Database Main registry (AHEAD Main). European Journal of Internal Medicine 2013; 24 (2): 151-60.

10. Purek L, Laule-Kilian K, Christ A, Klima T, Pfisterer M, Perruchoud A, Mueller C. Coronary artery disease and outcome in acute congestive heart failure. Heart 2006;92(5):598-602.

11. Nieminen MS, Brutsaert D, Dickstein $K$, Drexler $H$, Follath $F$, Harjola VP, et al. EuroHeart Survey Investigators; Heart Failure Association, European Society of Cardiology. EuroHeart Failure Survey II (EHFS II): a survey on hospitalized acute heart failure patients: description of population. Eur Heart J. 2006;27(22):2725-36.

12. Steg PG, Dabbous OH, Feldman LJ, CohenSolal A, Aumont MC, Lopez-Sendon J, et al. Determinants and prognostic impact of heart failure complicating acute coronary syndromes: observations from the Global Registry of Acute Coronary Events (GRACE). Circulation. 2004; 109(4):494-9.
13. AlFaleh $H$, Elasfar AA, Ullah $A$, AlHabib KF, Hersi $A$, Mimish $L$, et al. Acute heart failure with and without acute coronary syndrome: clinical correlates and prognostic impact (From the HEARTS registry). BMC CardiovasC Disord. 2016;16:98.

14. Tarvasmäki T, Harjola VP, Nieminen MS, Siirilä-Waris K, Tolonen J, Tolppanen $\mathrm{H}$, et al. FINN-AKVA Study Group. Acute heart failure with and without concomitant acute coronary syndromes: patient characteristics, management, and survival. J Card Fail. 2014;20(10):723-30.

15. Djousse L, Driver JA, Gaziano JM. Relation between modifiable lifestyle factors and lifetime risk of heart failure. JAMA 2009;302:394-400.

16. Maggioni AP, Dahlström U, Filippatos G, Chioncel O, Leiro MC, Drozdz J, et al. EURObservational Research Programme: regional differences and 1-year follow-up results of the Heart Failure Pilot Survey (ESC-HF Pilot). Eur J Heart Fail. 2013;15:808-17.

17. Crespo-Leiro MG, Anker SD, Maggioni AP, Coats AJ, Filippatos G, Ruschitzka $F$, et al. Heart Failure Association (HFA) of the European Society of Cardiology (ESC). European Society of Cardiology Heart Failure Long-Term Registry (ESC-HF-LT): 1-year follow-up outcomes and differences across regions. Eur J Heart Fail. 2016; 18(6):613-25.

18. Ammar KA, Jacobsen SJ, Mahoney DW, Kors JA, Redfield MM, Burnett JC Jr, et al. Prevalence and prognostic significance of heart failure stages: application of the American College of Cardiology/American Heart Association heart failure staging criteria in the community. Circulation. 2007; 115(12):1563-70.

19. Felker GM, Mentz RJ, Teerlink JR, Voors AA, Pang PS, Ponikowski $P$, et al. Serial high sensitivity cardiac troponin $T$ measurement in acute heart failure: insights from the RELAX-AHF study. Eur J Heart Fail. 2015;17 (12) :1262-70

20. O'Gara PT, Kushner FG, Ascheim DD, Casey DE Jr, Chung MK, de Lemos JA, et al. 2013 ACCF/AHA guideline for the management of ST-elevation myocardial infarction: executive summary: a report of the American College of Cardiology Foundation/American Heart Association Task Force on Practice Guidelines. J Am Coll Cardiol. 2013;61(4):485-510.

21. Amsterdam EA, Wenger NK, Brindis RG, Casey DE Jr, Ganiats TG, Holmes DR Jr, et al.2014 AHA/ACC Guideline for the Management of Patients with Non-STElevation Acute Coronary Syndromes: a report of the American College of Cardiology/American Heart Association Task Force on Practice Guidelines. J Am Coll Cardiol. 2014;64(24):e139-228. 
22. Roberts $E$, Ludman AJ, Dworzynski K, AlMohammad A, Cowie MR, McMurray JJV, et al. The diagnostic accuracy of the natriuretic peptides in heart failure: systematic review and diagnostic metaanalysis in the acute care setting. BMJ 2015;350:h910

23. Roffi M, Patrono C, Collet JP, Mueller C, Valgimigli M, Andreotti F, et al. 2015 ESC Guidelines for the Management of Acute Coronary Syndromes in Patients Presenting Without Persistent ST-segment Elevation. Rev Esp Cardiol. 2015;68(12):1125.e11125.e64

24. Konstantinides SV, Torbicki A, Agnelli G, Danchin N, Fitzmaurice D, Galiè N, et al. Task Force for the Diagnosis and Management of Acute Pulmonary Embolism of the European Society of Cardiology (ESC). 2014 ESC guidelines on the diagnosis and management of acute pulmonary embolism. Eur Heart J. 2014;35(43):3033-69.

25. Mebazaa A, Yilmaz MB, Levy P, Ponikowski $P$, Peacock WF, Laribi $S$, et al. Recommendations on pre-hospital \& early hospital management of acute heart failure: a consensus paper from the Heart Failure Association of the European Society of Cardiology, the European Society of Emergency Medicine and the Society of Academic Emergency Medicine. Eur J Heart Fail. 2015; 17(6):544-58.

26. Prins KW, Neill JM, Tyler JO, Eckman PM, Duval S. Effects of Beta-Blocker Withdrawal in Acute Decompensated Heart Failure: A Systematic Review and MetaAnalysis. JACC Heart Fail. 2015;3(8):64753.

27. Faris RF, Flather $M$, Purcell $H$, Poole-Wilson PA, Coats AJ. Diuretics for heart failure. Cochrane Database Syst Rev. 2012;2: CD003838.

28. King JB, Bress AP, Reese AD, Munger MA. Neprilysin inhibition in heart failure with reduced ejection fraction: a clinical review. Pharmacother J Hum Pharmacol Drug Ther. 2015;35 (9):823-37.

29. McMurray JJ, Packer M, Desai AS, Gong J, Lefkowitz MP, Rizkala AR, Rouleau J, Shi VC, Solomon SD, Swedberg K, Zile MR; PARADIGM-HF Committees and Investigators. Dual angiotensin receptor and neprilysin inhibition as an alternative to angiotensin-converting enzyme inhibition in patients with chronic systolic heart failure: rationale for and design of the Prospective comparison of ARNI with ACEI to Determine Impact on Global Mortality and morbidity in Heart Failure trial (PARADIGM-HF). Eur J Heart Fail. 2013;15(9):1062-73.

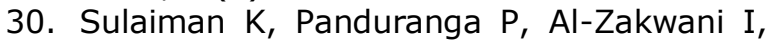
Alsheikh-Ali AA, AlHabib KF, Al-Suwaidi J, et al. Rationale, Design, Methodology and
Hospital Characteristics of the First Gulf Acute Heart Failure Registry (Gulf CARE). Heart Views. 2014;15(1):6-12.

31. Spinar J, Parenica J, Vitovec J, Widimsky P, Linhart A, Fedorco $M$, et al. Baseline characteristics and hospital mortality in the Acute Heart Failure Database (AHEAD) Main registry. Crit Care. 2011;15(6):R291.

32. Follath F, Yilmaz MB, Delgado JF, Parissis JT, Porcher R, Gayat E, et al. Clinical presentation, management and outcomes in the Acute Heart Failure Global Survey of Standard Treatment (ALARM-HF). Intensive Care Med. 2011;37(4):619-26.

33. Milazzo V, Cosentino N, Marenzi G. Extracorporeal ultrafiltration for acute heart failure: patient selection and perspectives. Vasc Health Risk Manag 2017;13: 449-56.

34. Greenberg G, Cohen E, Garty M, Iakobishvili Z, Sandach A, Behar S, et al. Outcomes of acute heart failure associated with acute coronary syndrome versus other causes. Acute Card Care. 2011;13(2):8792.

35. Shah RV, Holmes D, Anderson M, Wang TY, Kontos MC, Wiviott SD, Scirica BM. Risk of heart failure complication during hospitalization for acute myocardial infarction in a contemporary population: insights from the National Cardiovascular Data ACTION Registry. Circ Heart Fail. 2012;5(6):693-702.

36. Flaherty JD, Bax JJ, De Luca L, Rossi JS, Davidson CJ, Filippatos G, et al. Acute Heart Failure Syndromes International Working Group. Acute heart failure syndromes in patients with coronary artery disease early assessment and treatment. J Am Coll Cardiol. 2009;53(3):254-63.

37. Ferreira J. Vascular phenotypes of acute decompensated vs. new-onset heart failure: treatment implications. ESC Heart Failure 2017; 4 (4): 679-85.

38. Sulo G, Igland J, Nygård $O$, Vollset $S E$, Ebbing $M$, Poulter $N$, et al. Prognostic Impact of In-Hospital and Postdischarge Heart Failure in Patients With Acute Myocardial Infarction: A Nationwide Analysis Using Data From the Cardiovascular Disease in Norway (CVDNOR) Project. J Am Heart Assoc. 2017;6(3). pii: e005277.

39. Yancy CW, Jessup M, Bozkurt B, Butler J, Casey DE Jr, Drazner MH, et al. 2013 ACCF/AHA guideline for the management of heart failure: A report of the American College of Cardiology Foundation/American Heart Association Task Force on Practice Guidelines. J Am Coll Cardiol 2013;62 (16): e147-e239.

40. Zheng SL, Chan FT, Nabeebaccus AA, Shah AM, McDonagh T, Okonko DO, et al. Drug treatment effects on outcomes in heart failure with preserved ejection fraction: A 
systematic review and meta-analysis. Heart 2018; 104 (5): 407-15.

41. Fonarow GC, Stough WG, Abraham WT, Albert NM, Gheorghiade M, Greenberg BH, et al. Characteristics, treatments, and outcomes of patients with preserved systolic function hospitalized for heart failure: A report from the OPTIMIZE-HF Registry. J Am Coll Cardiol 2007; 50 (8): 768-77.

42. Yancy CW, Lopatin M, Stevenson LW, De Marco T, Fonarow GC. Clinical presentation, management, and in-hospital outcomes of patients admitted with acute decompensated heart failure with preserved systolic function: A report from the Acute Decompensated Heart Failure National Registry (ADHERE) Database. JAm Coll Cardiol 2006; 47(1):76-84.

43. Toma M, Ezekowitz JA, Bakal JA, O'Connor $C M$, Hernandez AF, Sardar MR, et al. The relationship between left ventricular ejection fraction and mortality in patients with acute heart failure: Insights from the ASCEND-HF Trial. Eur J Heart Fail 2014; 16 (3):334-41.

44. Kapoor JR, Kapoor R, Ju C, Heidenreich PA, Eapen ZJ, Hernandez AF, et al. Precipitating clinical factors, heart failure characterization, and outcomes in patients hospitalized with heart failure with reduced, borderline, and preserved ejection fraction. JACC Heart Fail 2016;4 (6): 464-72.

45. Cheng RK, Cox M, Neely ML, Heidenreich PA, Bhatt DL, Eapen ZJ, et al. Outcomes in patients with heart failure with preserved, borderline, and reduced ejection fraction in the Medicare population. Am Heart J 2014; 168 (5): 721-30.

46. Tsuji K, Sakata Y, Nochioka K, Miura M, Yamauchi T, Onose $T$, et al. Characterization of heart failure patients with mid-range left ventricular ejection fraction: A report from the CHART-2 Study. Eur J Heart Fail 2017; 19 (10): 1258-69.

47. Hsu JJ, Ziaeian B, Fonarow GC. Heart failure with mid-range (borderline) ejection fraction: Clinical implications and future directions. JACC Heart Fail 2017;5 (1):763-71.

48. Lund LH, Claggett B, Liu J, Lam CS, Jhund PS, Rosano GM, et al. Heart failure with mid-range ejection fraction in CHARM: Characteristics, outcomes and effect of candesartan across the entire ejection fraction spectrum. Eur J Heart Fail 2018;20 (8):1230-9.

49. Hernandez AF, Hammill BG, O'Connor CM, Schulman KA, Curtis LH, Fonarow GC. Clinical effectiveness of beta-blockers in heart failure: Findings from the OPTIMIZEHF (Organized Program to Initiate Lifesaving Treatment in Hospitalized Patients with Heart Failure) Registry. J Am Coll Cardiol 2009; 53 (2): 184-92.

50. Yamamoto K, Origasa H, Hori M. Effects of carvedilol on heart failure with preserved ejection fraction: The Japanese Diastolic Heart Failure Study (J-DHF). Eur J Heart Fail 2013; 15 (1): 110-8.

51. Gattis WA, O'Connor CM, Gallup DS, Hasselblad V, Gheorghiade M, IMPACT-HF Investigators and Coordinators. Predischarge initiation of carvedilol in patients hospitalized for decompensated heart failure. Am J Cardiol 2004;(43)9: 1534-41.

52. Jondeau G, Neuder $Y$, Eicher J-C, Jourdain $P$, Fauveau $E$, Galinier $M$, et al. BCONVINCED: Betablocker CONtinuation Vs. INterruption in patients with Congestive heart failure hospitalizED for a decompensation episode. Eur Heart J 2009; 30 (18): 2186-292. 